\title{
Magnetic flows on homogeneous spaces
}

\author{
Alexey V. Bolsinov* and Božidar Jovanović***
}

\begin{abstract}
We consider magnetic geodesic flows of the normal metrics on a class of homogeneous spaces, in particular adjoint orbits of compact Lie groups. We give the proof of the noncommutative integrability of flows and show, in addition, for the case of adjoint orbits, the usual Liouville integrability by means of analytic integrals. We also consider the potential systems on adjoint orbits, which are generalizations of the magnetic spherical pendulum. The complete integrability of such system is proved for an arbitrary adjoint orbit of a compact semisimple Lie group.
\end{abstract}

Mathematics Subject Classification (2000). 70H06, 37J35, 53D25.

Keywords. Magnetic flows, non-commutative integrability, compatible Poisson brackets.

\section{Introduction}

Let $Q$ be a smooth manifold with a Riemannian metric $g=\left(g_{i j}\right)$. Consider an arbitrary local coordinate system $x^{1}, \ldots, x^{n}$ and pass from velocities $\dot{x}^{i}$ to momenta $p_{j}$ by using the standard transformation $p_{j}=g_{i j} \dot{x}^{i}$. Then $x^{i}, p_{i}(i=1, \ldots, n)$ represent a local coordinate system on the cotangent bundle $T^{*} Q$ and the standard symplectic form on $T^{*} Q$ reads $\omega=\sum d p_{i} \wedge d x^{i}$. The corresponding canonical Poisson brackets are given by

$$
\{f, g\}_{0}=\sum_{i=1}^{n}\left(\frac{\partial f}{\partial x^{i}} \frac{\partial g}{\partial p_{i}}-\frac{\partial g}{\partial x^{i}} \frac{\partial f}{\partial p_{i}}\right) .
$$

The equations of the geodesic flow have the Hamiltonian form on $\left(T^{*} Q, \omega\right)$ :

$$
\frac{d f}{d t}=\{f, H\}_{0} \quad \Longleftrightarrow \quad \frac{d x^{i}}{d t}=\frac{\partial H}{\partial p_{i}}, \quad \frac{d p_{i}}{d t}=-\frac{\partial H}{\partial x^{i}},
$$

\footnotetext{
*Supported by RFBR 05-01-00978.

** Supported by the Serbian Ministry of Science, Project 144014 "Geometry and Topology of Manifolds and Integrable Dynamical Systems".
} 
where the Hamiltonian $H$ is

$$
H(x, p)=\frac{1}{2} \sum_{i, j=1}^{n} g^{i j} p_{i} p_{j}=\frac{1}{2} \sum_{i, j=1}^{n} g_{i j} \dot{x}^{i} \dot{x}^{j} .
$$

Here $g^{i j}$ are the coefficients of the tensor inverse to the metric.

The geodesic flow can be interpreted as the inertial motion of a particle on $Q$ with the kinetic energy given by (2). The motion of the particle under the influence of the additional magnetic field given by a closed 2 -form

$$
\Omega=\sum_{1 \leq i<j \leq n} F_{i j}(x) d x^{i} \wedge d x^{j},
$$

is described by the following equations:

$$
\frac{d x^{i}}{d t}=\frac{\partial H}{\partial p_{i}}, \quad \frac{d p_{i}}{d t}=-\frac{\partial H}{\partial x^{i}}+\sum_{j=1}^{n} F_{i j} \frac{\partial H}{\partial p_{j}} .
$$

The equations (3) are Hamiltonian with respect to the "twisted" symplectic form $\omega+\rho^{*} \Omega$, where $\rho: T^{*} Q \rightarrow Q$ is the natural projection. Namely, the new Poisson bracket is given by

$$
\{f, g\}=\{f, g\}_{0}+\sum_{i, j=1}^{n} F_{i j} \frac{\partial f}{\partial p_{i}} \frac{\partial g}{\partial p_{j}},
$$

and the Hamiltonian equations $\dot{f}=\{f, H\}$ read (3).

The flow (3) is called magnetic geodesic flow on the Riemannian manifold $(Q, g)$ with respect to the magnetic field $\Omega$. For simplicity, we shall refer to (4) as a magnetic Poisson bracket and to $\left(T^{*} Q, \omega+\rho^{*} \Omega\right)$ as a magnetic cotangent bundle.

Outline and results of the paper. Our work has been inspired by a recent paper by Efimov [15] in which he proved the non-commutative integrability of magnetic geodesic flows on adjoint orbits of compact Lie groups. We observed that using the approach developed in [7], [8] one can extend this result to a wider class of homogeneous spaces and construct, in many cases, complete algebras of commuting integrals. Besides, this technics turned out to be useful in the theory of integrable magnetic potential systems. Such systems on Stiefel and Grassmann manifolds of two-dimensional planes in $\mathbb{R}^{n}$ and complex projective spaces were studied in [30], [32].

In Section 2, we recall the concept of non-commutative integrability suggested by Mishchenko and Fomenko [25] and its relation with the Hamiltonian group action established in [7]. In Section 3 we introduce a class of homogeneous spaces $G / H$ 
admitting a natural $G$-invariant magnetic field. Briefly, this construction can be explained as follows. Let $G$ be a compact Lie group, $H$ its closed subgroup and $\mathfrak{h}$ and $\mathfrak{g}$ denote the Lie algebras of $H$ and $G$, respectively. Suppose that $a \in \mathfrak{h}$ is $H$-adjoint invariant. In particular, $H \subset G_{a}$, where $G_{a} \subset G$ is the $G$-adjoint isotropy group of $a$. Consider the adjoint orbit $\mathcal{O}(a)$ through $a$ endowed with the standard Kirillov-Kostant symplectic form $\Omega_{\mathrm{KK}}$ (we can naturally identify adjoint and coadjoint orbits by the use of $\operatorname{Ad}_{G}$-invariant scalar product on $\mathfrak{g}$ ). Then we have the canonical submersion of homogeneous spaces $\sigma: G / H \rightarrow G / G_{a} \cong \mathcal{O}(a)$ and the closed two-form $\Omega=\sigma^{*} \Omega_{\mathrm{KK}}$ gives us the required magnetic field on $G / H$. In particular, for $H=G_{a}$ we obtain an adjoint orbit with magnetic term being the Kirillov-Kostant form.

We prove the non-commutative integrability of geodesic magnetic flows of the normal metrics (Theorem 2) on $G / H$ and show, in addition, that for the case of adjoint orbits one can find enough commuting analytic integrals (Theorem 3 ). The proof is based on recent results concerning geodesic flows on homogeneous spaces [6], [8], [23].

In Section 4, we study the motion of a particle on coadjoint orbits under the influence of an additional potential force field. For the Lie algebra so(3), the system represents the magnetic spherical pendulum. The generalization of the magnetic spherical pendulum to the complex projective spaces is obtained in [32].

In Section 5, we give a representation of the system in the semidirect product $\mathfrak{g} \oplus_{\mathrm{ad}} \mathfrak{g}$ and, following the bi-Hamiltonian approach, prove its complete integrability for adjoint orbits of compact semisimple Lie groups (Corollary 2, Theorem 4). Various aspects of representations of (polarized) coadjoint orbits in semidirect products as magnetic cotangent bundles are studied in [26], [19], [13], [30], [2].

Let us emphasize that in the present paper we consider integrability as a qualitative phenomenon: the phase space of the system is foliated almost everywhere by isotropic invariant tori with quasiperiodic dynamics. Sometimes this property is not the same as the classical integrability, i.e., existence of explicit formulae for solutions. The simplest example that demonstrates the difference between two types of integrability is the magnetic geodesic flow on a compact constant negative curvature surface. We can represent the geodesics explicitly as projections of magnetic lines from the hyperbolic 2-plane (lines of constant geodesic curvature), but the restriction of the flow onto energy levels $H>h_{\mathrm{cr}}$ are Anosov flows, while the restriction onto energy levels $H<h_{\mathrm{cr}}$ are analytically integrable, where $h_{\mathrm{cr}}$ is some critical level of energy (e.g., see [17], [33]). 


\section{Integrable systems related to Hamiltonian actions}

There are a lot of examples of integrable Hamiltonian systems with $n$ degrees of freedom that admit more than $n$ (noncommuting) integrals. Then, under some assumptions, the $n$ dimensional Lagrangian tori are foliated by lower dimensional isotropic tori. This happens in the case of the so-called non-commutative integrability studied by Nekhoroshev [27] and Mishchenko and Fomenko [25] (see also [7], [35]).

Let $M$ be a Poisson manifold and $\mathcal{F}$ be a Poisson subalgebra of $C^{\infty}(M)$. Suppose that in the neighborhood of a generic point $x$ we can find exactly $l$ independent functions $f_{1}, \ldots, f_{l} \in \mathcal{F}$ and the corank of the matrix $\left\{f_{i}, f_{j}\right\}$ is equal to some constant $r$. Then numbers $l$ and $r$ are called differential dimension and differential index of $\mathcal{F}$ and they are denoted by $\operatorname{ddim} \mathcal{F}$ and $\operatorname{dind} \mathcal{F}$, respectively. The algebra $\mathcal{F}$ is called complete if:

$$
\operatorname{ddim} \mathcal{F}+\operatorname{dind} \mathcal{F}=\operatorname{dim} M+\operatorname{corank}\{\cdot, \cdot\},
$$

If $\mathcal{F}$ is any algebra of functions, then we shall say that $\mathcal{A} \subset \mathcal{F}$ is a complete subalgebra if

$$
\operatorname{ddim} \mathcal{A}+\operatorname{dind} \mathcal{A}=\operatorname{ddim} \mathcal{F}+\operatorname{dind} \mathcal{F} .
$$

The Hamiltonian system $\dot{x}=X_{H}(x)$ is completely integrable in the non-commutative sense if it possesses a complete algebra of first integrals $\mathcal{F}$. Then (under compactness condition) $M$ is almost everywhere foliated by $(\operatorname{dind} \mathcal{F}-\operatorname{corank}\{\cdot, \cdot\})$-dimensional invariant isotropic tori. Similarly as in the Liouville theorem, the tori are filled up with quasi-periodic trajectories.

Mishchenko and Fomenko stated the conjecture that non-commutative integrable systems $\dot{x}=X_{H}(x)$ are integrable in the usual commutative sense by means of integrals from $\mathcal{A}$ that belong to the same functional class as the original non-commutative algebra of integrals. The conjecture is proved in $C^{\infty}$-smooth case [7]. In the analytic case, when $\mathcal{F}=\operatorname{span}_{\mathbb{R}}\left\{f_{1}, \ldots, f_{l}\right\}$ is a finite-dimensional Lie algebra, the conjecture has been proved by Mishchenko and Fomenko in the semisimple case and just recently by Sadetov [31] for arbitrary Lie algebras.

Now, let a connected compact Lie group $G$ act on a $2 n$-dimensional connected symplectic manifold $(M, \omega)$. Suppose the action is Hamiltonian with the momentum mapping $\Phi: M \rightarrow \mathfrak{g}^{*} \cong \mathfrak{g}\left(\mathfrak{g}^{*}\right.$ is the dual space of the Lie algebra $\mathfrak{g}$, we use the identification by means of $\operatorname{Ad}_{G}$-invariant scalar product $\langle\cdot, \cdot\rangle$ on $\mathfrak{g}$ ).

Consider the following two natural classes of functions on $M$. Let $\mathcal{F}_{1}$ be the set of functions in $C^{\infty}(M)$ obtained by pulling-back the algebra $C^{\infty}(\mathfrak{g})$ by the moment map $\mathcal{F}_{1}=\Phi^{*} C^{\infty}(\mathfrak{g})$. Let $\mathcal{F}_{2}$ be the set of $G$-invariant functions in $C^{\infty}(M)$. The mapping $f \mapsto f \circ \Phi$ is a morphism of Poisson structures:

$$
\{f \circ \Phi, g \circ \Phi\}(x)=\{f, g\}_{\mathfrak{g}}(\eta), \quad \eta=\Phi(x),
$$


where $\{\cdot, \cdot\}_{\mathfrak{g}}$ is the Lie-Poisson bracket on $\mathfrak{g}$ :

$$
\{f, g\}_{\mathfrak{g}}(\eta)=\langle\eta,[\nabla f(\eta), \nabla g(\eta)]\rangle, \quad f, g: \mathfrak{g} \rightarrow \mathbb{R} .
$$

Thus, $\mathscr{F}_{1}$ is closed under the Poisson bracket. Since $G$ acts in a Hamiltonian way, $\mathcal{F}_{2}$ is closed under the Poisson bracket as well. The second essential fact is that $h \circ \Phi$ commute with any $G$-invariant function (the Noether theorem). In other words: $\left\{\mathcal{F}_{1}, \mathscr{F}_{2}\right\}=0$.

The following theorem, although it is a reformulation of some well-known facts about the momentum mapping (e.g., see [16]), is fundamental in the considerations below (see [7] for more details).

Let $\mathcal{A} \subset C^{\infty}(\mathfrak{g})$ be a Lie subalgebra and $\Phi^{*} \mathcal{A}=\{h \circ \Phi, h \in \mathcal{A}\}$ the pull-back of $\mathcal{A}$ by the momentum mapping. Then we have:

Theorem 1. (i) The algebra of functions $\mathcal{F}_{1}+\mathcal{F}_{2}$ is complete:

$$
\operatorname{ddim}\left(\mathcal{F}_{1}+\mathcal{F}_{2}\right)+\operatorname{dind}\left(\mathcal{F}_{1}+\mathcal{F}_{2}\right)=\operatorname{dim} M
$$

The dimension of regular invariant isotropic tori, common level sets of functions from $\mathcal{F}_{1}+\mathcal{F}_{2}$, is equal to

$$
\operatorname{dim} G_{\mu}-\operatorname{dim} G_{x},
$$

for generic $x \in M, \mu=\Phi(x)\left(G_{\mu}\right.$ and $G_{x}$ denotes the isotropy groups of $G$ action at $\mu$ and $x$ ).

(ii) $\Phi^{*} \mathcal{A}+\mathcal{F}_{2}$ is a complete algebra on $M$ if and only if $\mathcal{A}$ is a complete algebra on a generic adjoint orbit $\mathcal{O}(\mu) \subset \Phi(M)$.

(iii) If $\mathcal{B}$ is complete (commutative) subalgebra of $\mathcal{F}_{2}$ and $\mathcal{A}$ is complete (commutative) algebra on the orbit $\mathcal{O}(\mu)$, for generic $\mu \in \Phi(M)$ then $\Phi^{*} \mathcal{A}+\mathscr{B}$ is complete (commutative) algebra on $M$.

Notice that instead of commutative subalgebras one usually consider sets of commuting functions. Clearly, each commutative set generates a certain commutative subalgebra. The notions of completeness, ddim and dind for a commutative set are defined just in the same way as above.

\section{Magnetic geodesic flows}

Let $G$ be a compact connected Lie group with the Lie algebra $\mathfrak{g}=T_{e} G$. Let us fix some bi-invariant metric $d s_{0}^{2}$ on $G$, i.e., $\operatorname{Ad}_{G}$-invariant scalar product $\langle\cdot, \cdot\rangle$ on $\mathfrak{g}$. We can identify $\mathfrak{g}^{*}$ and $\mathfrak{g}$ by $\langle\cdot, \cdot\rangle$.

Consider an arbitrary homogeneous space $G / H$ of the Lie group $G$. The metric $d s_{0}^{2}$ induces so called normal metric on $G / H$. We shall denote the normal metric 
also by $d s_{0}^{2}$. By the use of $d s_{0}^{2}$ we identify $T^{*} G \cong T G$ and $T^{*}(G / H) \cong T(G / H)$. Let $\mathfrak{h}$ be the Lie algebra of $H$ and $\mathfrak{g}=\mathfrak{h}+\mathfrak{v}$ the orthogonal decomposition. Then $\mathfrak{v}$ can be naturally identified with $T_{\pi(e)}(G / H)$ and $T_{\pi(e)}^{*}(G / H)$, where $\pi: G \rightarrow G / H$ is the canonical projection.

Construction of the magnetic field. We introduce a class of homogeneous spaces $G / H$ having a natural construction of the magnetic term, consisting of pairs $(G, H)$, where $H$ have one-point adjoint orbits. Let $a \in \mathfrak{h}$ be the $H$-adjoint invariant. Then $H$ is a subgroup of the $G$-adjoint isotropy group $G_{a}$ of $a$. The adjoint orbit $\mathcal{O}(a)$ through $a$ carries the Kirillov-Kostant symplectic form $\Omega_{\mathrm{KK}}$. Then we have canonical submersion of homogeneous spaces

$$
\sigma: G / H \rightarrow G / G_{a} \cong \mathcal{O}(a)
$$

and the closed two-form $\Omega=\sigma^{*} \Omega_{\mathrm{KK}}$ gives us the required magnetic field on $G / H$.

The form $\Omega$ is $G$-invariant. From the definition of $\Omega_{\mathrm{KK}}$ (see equation (10)) one can easily prove that at the point $\pi(e), \Omega$ is given by

$$
\left.\Omega\left(\xi_{1}, \xi_{2}\right)\right|_{\pi(e)}=-\left\langle a,\left[\xi_{1}, \xi_{2}\right]\right\rangle, \quad \xi_{1}, \xi_{2} \in \mathfrak{v} \cong T_{\pi(e)}(G / H)
$$

Below we give another natural description of the form $\Omega$.

Reduction. Consider the right action of the Lie subgroup $H$ to $G:(g, h) \mapsto g h$, $g \in G, h \in H$, and extend it to the right Hamiltonian action on $T^{*} G$. After identification $\mathfrak{h} \cong \mathfrak{h}^{*}$, we get the momentum mapping

$$
\Psi: T^{*} G \rightarrow \mathfrak{h}, \quad \Psi(g \cdot \xi)=\operatorname{pr}_{\mathfrak{h}} \xi, \quad \xi \in \mathfrak{g} .
$$

Here $\mathrm{pr}_{\mathfrak{h}}$ denotes the orthogonal projection with respect to the invariant scalar product $\langle\cdot, \cdot\rangle$.

It is well known that the symplectic reduced space $\Psi^{-1}(0) / H$ is symplectomorphic to $\left(T^{*}(G / H), \omega\right)$, where $\omega$ is the canonical symplectic form on $T^{*}(G / H)$. On the other side, the reduced spaces $\Psi^{-1}(a) / H_{a}$, are diffeomorphic to the fibre bundles over $T^{*}(G / H)$ with fibres being the $H$-adjoint orbit through $a$. In particular, if we deal with one-point orbit $\mathcal{O}_{H}(a)=\{a\}$, then the reduced space is symplectomorphic to the magnetic cotangent bundle of $G / H$ (see [1], [28]). Note that for connected $H$, $a$ is a $H$-adjoint invariant if and only if $a$ belongs to the center of $\mathfrak{h}$.

Proposition 1. Let $a \in \mathfrak{h}$ be the $H$-adjoint invariant and let $\epsilon$ be a real parameter. Then the symplectic reduced space $\Psi^{-1}(\epsilon a) / H$ is symplectomorphic to the magnetic cotangent bundle $T^{*}(G / H)$ endowed with the symplectic form $\omega+\epsilon \rho^{*} \Omega$. The form $\Omega$ is $G$-invariant and at the point $\pi(e)$ is given by (5). 
Proof. We only need to describe the magnetic term for $\epsilon=1$. Take a principal connection $\alpha$ on the $H$-bundle $G \rightarrow G / H$, that is a $\mathfrak{h}$-valued 1 -form with the property that the distribution $D=\operatorname{ker} \alpha \subset T G$ (horizontal distribution) is $H$-invariant and transversal to the orbit of $H$-action. For example we can take $\alpha$ such that $D$ is orthogonal to $H$-orbits with respect to the fixed bi-invariant metric. Then $\alpha_{g}(X)=$ $\operatorname{pr}_{\mathfrak{h}}\left(g^{-1} \cdot X\right)$ and $D_{g}=g \cdot \mathfrak{v}$. Define the 1 -form $\alpha_{a}=\langle\alpha, a\rangle$ on $G$. The magnetic form $\Omega$ is the unique 2 -form determined by (see Kummer [20])

$$
d \alpha_{a}=\pi^{*} \Omega \text {. }
$$

Since $\alpha$ is a connection and $a$ is $H$-invariant, the form $\Omega$ is well defined. Also, since $\pi$ is a submersion, $\Omega$ is closed but need not be exact.

For a vector $X \in T_{g} G$ we have the unique decomposition $X=X^{h}+X^{v}$, into the horizontal part $X^{h} \in g \cdot \mathfrak{v}$ and the vertical part $X^{v} \in g \cdot \mathfrak{h}$. The alternative description of the magnetic term is

$$
\Omega_{\pi(g)}(X, Y)=\left\langle a, \beta_{g}\left(X^{h}, Y^{h}\right)\right\rangle, \quad X, Y \in T_{\pi(g)}(G / H)
$$

where $X^{h}, Y^{h} \in T_{g} G$ are horizontal lifts of $X$ and $Y$ and $\beta$ is the curvature of the connection (h-valued 2-form on $G$ ).

Let $\bar{X}^{h}, \bar{Y}^{h}$ be arbitrary extensions of $X^{h}, Y^{h}$ to horizontal vector fields. Then

$$
\beta\left(X^{h}, Y^{h}\right)=-\alpha\left(\left[\bar{X}^{h}, \bar{Y}^{h}\right]\right)
$$

where $[\cdot, \cdot]$ is the commutator of vector fields. Now, by taking the left-invariant extensions of $X^{h}$ and $Y^{h}$ we come to the expression

$$
\beta\left(X^{h}, Y^{h}\right)=-\operatorname{pr}_{\mathfrak{h}}\left[g^{-1} \cdot X^{h}, g^{-1} \cdot Y^{h}\right],
$$

where $[\cdot, \cdot]$ is the Lie algebra commutator.

The horizontal lifts of $\xi_{1}, \xi_{2} \in \mathfrak{v} \cong T_{\pi(e)}(G / H)$ to $\mathfrak{g} \cong T_{e} G$ are exactly $\xi_{1}$ and $\xi_{2}$ considered as elements of $\mathfrak{g}$. Whence, from relations (6) and (7) we get the required expression (5) for the magnetic form $\Omega$ at $\pi(e)$.

Note that the above magnetic cotangent bundles of the homogeneous spaces naturally appear in the symplectic induction procedure over a point (see [13]).

The natural left $G$-action on $T^{*} G$ commutes with the right $H$-action and leaves the preimage $\Psi^{-1}(\epsilon a)$ invariant. Thus, from the well-known formula for the momentum mapping of the left $G$-action on $T^{*} G$ we get

Lemma 1. The momentum mapping

$$
\Phi_{\epsilon}: T^{*}(G / H) \rightarrow \mathfrak{g},
$$

of the natural $G$-action on $T^{*}(G / H)$ with respect to the symplectic form $\omega+\epsilon \rho^{*} \Omega$ is given by

$$
\Phi_{\epsilon}(g \cdot \eta)=\operatorname{Ad}_{g}(\eta+\epsilon a), \quad \eta \in \mathfrak{v}, g \cdot \eta \in T_{\pi(g)}^{*}(G / H) .
$$


Magnetic geodesic flows of normal metrics. The Hamiltonian function of the geodesic flow of the normal metric $d s_{0}^{2}$ is simply given by

$$
H_{0}=\frac{1}{2}\left\langle\Phi_{0}, \Phi_{0}\right\rangle
$$

Now, let $\mathcal{F}_{1}^{\epsilon}$ be the algebra of all analytic, polynomial in momenta, functions of the form

$$
\mathcal{F}_{1}^{\epsilon}=\left\{p \circ \Phi_{\epsilon}, p \in \mathbb{R}[\mathfrak{g}]\right\}
$$

and $\mathcal{F}_{2}$ be the algebra of all analytic, polynomial in momenta, $G$-invariant functions on $T^{*}(G / H)$. Then

$$
\left\{\mathcal{F}_{1}^{\epsilon}, \mathcal{F}_{2}\right\}_{\epsilon}=0,
$$

where $\{\cdot, \cdot\}_{\epsilon}$ are magnetic Poisson bracket with respect to $\omega+\epsilon \rho^{*} \Omega$.

Consider the Hamiltonian $H_{\epsilon}=\frac{1}{2}\left\langle\Phi_{\epsilon}, \Phi_{\epsilon}\right\rangle \in \mathcal{F}_{1}^{\epsilon}$. We have

$$
\begin{aligned}
H_{\epsilon}(g \cdot \eta) & =\frac{1}{2}\left\langle\operatorname{Ad}_{g} \eta, \operatorname{Ad}_{g} \eta\right\rangle+\epsilon\left\langle\operatorname{Ad}_{g} \eta, \operatorname{Ad}_{g} a\right\rangle+\epsilon^{2} \frac{1}{2}\left\langle\operatorname{Ad}_{g} a, \operatorname{Ad}_{g} a\right\rangle \\
& =H_{0}(g \cdot \eta)+\epsilon^{2} \frac{1}{2}\langle a, a\rangle=H_{0}(g \cdot \eta)+\mathrm{const},
\end{aligned}
$$

where we used that $\eta \in \mathfrak{v}$ is orthogonal to $a \in \mathfrak{h}$. Thus, we see that Hamiltonian flows of $H_{0}$ and $H_{\epsilon}$ coincides: $\dot{f}=\left\{f, H_{0}\right\}_{\epsilon}=\left\{f, H_{\epsilon}\right\}_{\epsilon}$. Since $H_{\epsilon}$ belongs to $\mathcal{F}_{1}^{\epsilon}$ its commutes with $\mathcal{F}_{2}$. On the other side, as a composition of the momentum mapping with an invariant polynomial, the function $H_{\epsilon}$ is also $G$-invariant and commutes with $\mathcal{F}_{1}^{\epsilon}$. Hence $\left\{H_{0}, \mathcal{F}_{1}^{\epsilon}+\mathcal{F}_{2}\right\}_{\epsilon}=0$.

From the above consideration and Theorem 1 we get the following result. Let, as before, $G$ be a compact Lie group and $H \subset G$ be a closed subgroup such that $\operatorname{Ad}_{H} a=a$ for a certain element $a \in \mathfrak{h} \subset \mathfrak{g}$.

Theorem 2. The magnetic geodesic flow of the normal metrics $d s_{0}^{2}$ on the homogeneous space $G / H$ with respect to the closed 2-form $\epsilon \Omega$ given by (5) is completely integrable in the non-commutative sense. The complete algebra of first integrals is $\mathcal{F}_{1}^{\epsilon}+\mathcal{F}_{2}$

Remark 1. The magnetic geodesic flow can be seen also as the reduction of the geodesic flow of the bi-invariant metric $d s_{0}^{2}$ from the invariant subspace $\Psi^{-1}(\epsilon a)$ to $\Psi^{-1}(\epsilon a) / H \cong T^{*}(G / H)$. With the above notation, this means that each magnetic geodesic line on $G / H$ is the projection of a certain geodesic $\gamma(t) \subset G$

$$
\gamma(t)=g_{0} \cdot \exp ((\xi+\epsilon a) t), \quad t \in \mathbb{R},
$$

where $\xi \in \mathfrak{o}$ and $g_{0}$ is the initial position. In such a way, the integrability of the magnetic geodesic flow can be also studied from the point of view of the symplectic 
reduction (see [18], [35]). More precisely, the reduction of the normal geodesic system from $T^{*} G$ to the Poisson manifold $\left(T^{*} G\right) / H$ is completely integrable in non-commutative sense (see Zung [35]). Since the symplectic leaves in $\left(T^{*} G\right) / H$ are Marsden-Weinstein reduced spaces, it appears that the symmetry reduction for a generic value of the momentum map $\Psi$ yields a system which is integrable in the non-commutative sense. The interpretation of these reduced systems in terms of the Yang-Mills analogue of the Lorentz force is well known (e.g., see [16]). The magnetic bundle $\Psi^{-1}(\epsilon a) / H$, in general, corresponds to a singular leaf in $\left(T^{*} G\right) / H$ and in this case some complementary work has to be given to prove the integrability.

Magnetic geodesic flows on adjoint orbits. Consider the (co)adjoint action of $G$ and the $G$-orbit $\mathcal{O}(a)$ through an element $a \in \mathfrak{g}$. In what follows, we shall use the representation of the adjoint orbit as a homogeneous space $G / H$, where $H=G_{a}$ is the isotropy group of $a$. Since $G$ is a compact connected Lie group, $G_{a}$ is also connected (e.g, see [16], page 259). We have

$$
\operatorname{ann}(a)=\{\xi \in \mathfrak{g},[\xi, a]=0\}=T_{e} G_{a} .
$$

By definition, the Kirillov-Kostant symplectic form $\Omega_{\mathrm{KK}}$ on $G / G_{a}$ is a $G$ invariant form, given at the point $\pi(e) \in G / G_{a}$ by

$$
\left.\Omega_{\mathrm{KK}}\left(\xi_{1}, \xi_{2}\right)\right|_{\pi(e)}=-\left\langle a,\left[\xi_{1}, \xi_{2}\right]\right\rangle, \quad \xi_{1}, \xi_{2} \in \operatorname{ann}(a)^{\perp}=[a, \mathfrak{g}],
$$

where $\xi_{1}, \xi_{2}$ are considered as tangent vectors to the orbit at $\pi(e)$. It follows from Proposition 1 that the Kirillov-Kostant form can be seen as a magnetic form obtained after right symplectic $G_{a}$-reduction of $T^{*} G$ as well.

From Theorem 2 we recover the Efimov result [15] (see also [9]):

Corollary 1. Let $G$ be a compact Lie group and $a \in \mathfrak{g}$. The magnetic geodesic flows of normal metric $d s_{0}^{2}$ on the (co)adjoint orbit $\mathcal{O}(a)=G / G_{a}$ with respect to the magnetic form $\epsilon \Omega_{\mathrm{KK}}$ is completely integrable in the non-commutative sense.

Remark 2. For a generic $\eta \in \operatorname{ann}(a)^{\perp}$ we have equality $\operatorname{dim} G_{\eta+\epsilon a}=\operatorname{dim} G_{\eta}$ for all $\epsilon \in \mathbb{R}$ (see [8], [23]). It implies that the dimension of the regular invariant tori does not depend on $\epsilon$ and is equal to

$$
\operatorname{dind}\left(\mathcal{F}_{1}^{\epsilon}+\mathscr{F}_{2}\right)=\operatorname{dim} G_{\eta}-\operatorname{dim}\left(G_{a}\right)_{\eta},
$$

for a generic element $\eta \in \operatorname{ann}(a)^{\perp}$ (see [6]). Therefore, the influence of the magnetic fields $\epsilon \Omega_{\mathrm{KK}}, \epsilon \in \mathbb{R}$ reflects as a deformation of the foliation of the phase space $\left(T^{*} \mathcal{O}(a), \omega+\epsilon \rho^{*} \Omega_{\mathrm{KK}}\right)$ by invariant isotropic tori. As the magnetic field increases, the magnetic geodesic lines become more curved. 
Example 1. On the unit round sphere (see the next section), the magnetic geodesic lines are circles on the sphere. It can be easily proved that for the motion with unit velocity, the radius of the circles is equal to $r_{\epsilon}=\operatorname{arctg}\left(\frac{1}{|\epsilon|}\right)$. As $|\epsilon|$ tends to infinity, $r_{\epsilon}$ tends to zero, and as $\epsilon$ tends to zero, then $r_{\epsilon}$ tends to $\frac{\pi}{2}$.

Commutative integrability. To prove the commutative integrability, we use the argument shift method developed by Mischenko and Fomenko [24] as a generalization of Manakov's construction [21].

Let $\mathbb{R}[\mathfrak{g}]^{G}$ be the algebra of $\operatorname{Ad}_{G}$-invariant polynomials on $\mathfrak{g}$. Then the polynomials

$$
\mathcal{A}_{c}=\left\{p(\cdot+\lambda c), \lambda \in \mathbb{R}, \quad p \in \mathbb{R}[\mathfrak{g}]^{G}\right\}
$$

obtained from the invariants by shifting the argument are in involution with respect to the Lie-Poisson bracket [24]. Furthermore, for every adjoint orbit in $\mathfrak{g}$, one can find $c \in \mathfrak{g}$, such that $\mathcal{A}_{c}$ is a complete commutative set of functions on this orbit. For regular orbits it is shown in [24]. For singular orbits there are several different proofs, see [22], [11], [5]. Thus, the argument shift method allows us to construct a complete commutative subalgebra in $\mathcal{F}_{1}^{\epsilon}$.

The $G$-invariant, polynomial in momenta functions on $T^{*}(G / H)$ are in one-toone correspondence with $\mathrm{Ad}_{H}$-invariant polynomials on $\mathfrak{v}$, via their restrictions to $T_{\pi}^{*}(e)(G / H) \cong \mathfrak{v}$. Within this identification, from (4), (5) and Thimm's formula for $\epsilon=0$ [34], the magnetic Poisson bracket $\{\cdot, \cdot\}_{\epsilon}$ on $T^{*}(G / H)$ corresponds to the following bracket on $\mathbb{R}[\mathfrak{v}]^{H}$

$$
\{f, g\}_{\mathfrak{v}}^{\epsilon}(\eta)=-\langle\eta+\epsilon a,[\nabla f(\eta), \nabla g(\eta)]\rangle, \quad f, g \in \mathbb{R}[\mathfrak{v}]^{H},
$$

where $\mathbb{R}[\mathfrak{v}]^{H}$ denotes the algebra of $\operatorname{Ad}_{H}$-invariant polynomials on $\mathfrak{v}$.

It is interesting that

$$
\Lambda_{\lambda_{1}, \lambda_{2}}=\lambda_{1}\{\cdot, \cdot\}_{\mathfrak{v}}^{0}+\lambda_{2}\{\cdot, \cdot\}_{\mathfrak{v}}^{a}, \quad \lambda_{1}^{2}+\lambda_{2}^{2} \neq 0
$$

is a pencil of the compatible Poisson brackets on $\mathbb{R}[\mathfrak{v}]^{H}$. Here

$$
\{f, g\}_{\mathfrak{v}}^{a}(\eta)=-\langle a,[\nabla f(\eta), \nabla g(\eta)]\rangle, \quad f, g \in \mathbb{R}[\mathfrak{v}]^{H} .
$$

By the use of the pencil (14) and the completeness criterion derived in [5], for the case of adjoint orbits, i.e, when $H=G_{a}$ and $\mathfrak{v}=\operatorname{ann}(a)^{\perp}$ one can conclude that the collection of Casimir functions of all the brackets $\Lambda_{1, \lambda}, \lambda \in \mathbb{R}$ :

$$
\mathcal{B}_{a}=\left\{p_{a}^{\lambda}(\eta)=p(\eta+\lambda a), \lambda \in \mathbb{R}, p \in \mathbb{R}[\mathfrak{g}]^{G}, \eta \in \operatorname{ann}(a)^{\perp}\right\}
$$

is a complete commutative subset in $\mathbb{R}\left[\operatorname{ann}(a)^{\perp}\right]^{G_{a}}$ with respect to the canonical bracket $\Lambda_{1,0}$. If $a$ is regular in $\mathfrak{g}$ then $G_{a}$ is a maximal torus. In this case the completeness of $\mathscr{B}_{a}$ can be easily verified (e.g., see [6], [8]). A nontrivial generalization to 
singular orbits of classical groups is done in [8], [12] and by Mykytyuk and Panasyuk for a general case [23]. Namely, it is proved that all (complexified) brackets $\Lambda_{\lambda_{1}, \lambda_{2}}$ have the same corank in a generic point $\eta \in \operatorname{ann}(a)^{\perp}$, equal to (11). It follows from Theorem 1.1 [5] that (16) is a complete commutative algebra with respect to each Poisson bracket (14) as well.

Whence, according Theorem 1, we get the following statement

Theorem 3. The magnetic geodesic flows of the normal metric $d s_{0}^{2}$ on the orbit $\mathcal{O}(a)=G / G_{a}$, with respect to the magnetic field $\epsilon \Omega_{\mathrm{KK}}$ is completely integrable in the commutative sense, by means of analytic, polynomial in momenta first integrals $\Phi_{\epsilon}^{*}\left(\mathcal{A}_{c}\right)+\mathscr{B}_{a}$, where $\mathcal{A}_{c}$ and $\mathcal{B}_{a}$ are given by (12) and (16), respectively.

The commutative integrability of the magnetic flows on the complex projective spaces is proved by Efimov in [14] (since the complex projective spaces are symmetric spaces, in this case the algebra of $\mathcal{F}_{2}$ is commutative).

The integrals $\mathcal{B}_{a}$ can be used for deforming the normal metric to a certain class of $G$-invariant metrics on $\mathcal{O}(a)$ with completely integrable magnetic geodesic flows. Theorem 3 is announced in [9], where one can find the explicit description of the deformed flows within the standard representation of the orbit $\mathcal{O}(a)$, as a submanifold of $\mathfrak{g}$.

Remark 3. If $H$ is a subgroup of the isotropy group $G_{a}$, then the rank of the bracket $\Lambda_{0,1}$, in general, is smaller then the rank of the other brackets from the pencil. Then Theorem 1.1 [5] implies that Casimir functions of the brackets $\Lambda_{1, \lambda}, \lambda \in \mathbb{R}$ do not form a complete set with respect to the magnetic bracket (13). In order to get complete commutative algebra in $\left(\mathbb{R}[\mathfrak{v}]^{H}, \Lambda_{1, \epsilon}\right)$, one has to find enough additional commutative functions among Casimirs of $\Lambda_{0,1}$.

\section{Magnetic pendulum on adjoint orbits}

From now on, we shall consider the orbit $\mathcal{O}(a)$ realised as a submanifold of $\mathfrak{g}$. In this representation, the geometry of Hamiltonian flows on $T^{*} \mathcal{O}(a)$ is studied by Bloch, Brockett and Crouch [3], while $G$-invariant magnetic geodesic flows are studied in [9].

The tangent space at $x=\operatorname{Ad}_{g}(a)$ is simply the orthogonal complement to ann $(x)$. Consider the cotangent bundle $T^{*} \mathcal{O}(a)$ as a submanifold of $\mathfrak{g} \times \mathfrak{g}$ :

$$
T^{*} \mathcal{O}(a)=\left\{(x, p) \mid x=\operatorname{Ad}_{g}(a), p \in \operatorname{ann}(x)^{\perp}\right\},
$$

with the paring between $p \in T_{x}^{*} \mathcal{O}(a) \cong \operatorname{ann}(x)^{\perp}$ and $\eta \in T_{x} \mathcal{O}(a)$ given by $p(\eta)=$ $\langle p, \eta\rangle$. Then the canonical symplectic form $\omega$ on $T^{*} \mathcal{O}(a)$ can be seen as a restriction 
of the canonical linear symplectic form of the ambient space $\mathfrak{g} \times \mathfrak{g}: \sum_{i=1}^{\operatorname{dim} \mathfrak{g}} d p_{i} \wedge d x_{i}$, where $p_{i}, x_{i}$ are coordinates of $p$ and $x$ with respect to some base of $\mathfrak{g}$.

Let $\xi \in \mathfrak{g}$ and $x=\operatorname{Ad}_{g}(a)$. Since $\xi_{x}=\left.\frac{d}{d s} \operatorname{Ad}_{\exp (s \xi)}(x)\right|_{s=0}=[\xi, x]$, the momentum mapping of the Hamiltonian $G$-action

$$
g \cdot(x, p)=\left(\operatorname{Ad}_{g} x, \operatorname{Ad}_{g} p\right)
$$

on $\left(T^{*} \mathcal{O}(a), \omega\right)$ is given by the relation $\left\langle\Phi_{0}(x, p), \xi\right\rangle=\left\langle p, \xi_{x}\right\rangle=\langle p,[\xi, x]\rangle$. That is,

$$
\Phi_{0}(x, p)=[x, p] .
$$

Therefore, the momentum mapping (8), for $\epsilon \neq 0$, and normal metric Hamiltonian read

$$
\begin{aligned}
& \Phi_{\epsilon}(x, p)=[x, p]+\epsilon x, \\
& H_{0}(x, p)=\frac{1}{2}\langle[x, p],[x, p]\rangle .
\end{aligned}
$$

Magnetic spherical pendulum. As an example, consider the Lie group $\mathrm{SO}(3)$. The Lie algebra $s o(3)$ is isomorphic to the Euclidean space $\mathbb{R}^{3}$ with bracket operation being the standard vector product. The adjoint orbits are spheres $\langle\vec{x}, \vec{x}\rangle=$ const. Let us consider the unit sphere $S^{2}$ and its cotangent bundle $T^{*} S^{2}$ realized as a submanifold of $\mathbb{R}^{6}$ :

$$
T^{*} S^{2}=\left\{(\vec{x}, \vec{p}) \in \mathbb{R}^{6} \mid \phi_{1}=\langle\vec{x}, \vec{x}\rangle=1, \phi_{2}=\langle\vec{x}, \vec{p}\rangle=0\right\} .
$$

The momentum mapping of the natural $\mathrm{SO}(3)(3)$ action on $\left(T^{*} S^{2}, \omega\right)$ is $\Phi_{0}=$ $\vec{x} \times \vec{p}$ and the Hamiltonian of the normal metric $d s_{0}^{2}$ reduces to $H=\frac{1}{2}\langle\vec{p}, \vec{p}\rangle$. This is the kinetic energy of the unit mass particle motion on the sphere. Adding the magnetic term $\epsilon \rho^{*} \Omega_{\mathrm{KK}}$ to the canonical form represents the influence of the magnetic monopole with the force equal to $\epsilon \vec{x} \times \vec{p}$. It is well known that two famous integrable potential systems on the sphere remain integrable after including the magnetic monopole, namely the spherical pendulum and the Neumann system. Let us consider the spherical pendulum. Then the Hamiltonian of the system becomes:

$$
H=\frac{1}{2}\langle\vec{p}, \vec{p}\rangle-\kappa\langle\vec{b}, \vec{x}\rangle,
$$

where $\vec{b}$ is a constant unit vector. The equations of the motion of the particle with the energy (18) under the influence of the magnetic force $\epsilon \vec{x} \times \vec{p}$, in the redundant variables $(x, p)$ are

$$
\frac{d}{d t} \vec{x}=p, \quad \frac{d}{d t} \vec{p}=\kappa \vec{b}+\epsilon \vec{x} \times \vec{p}+\lambda \vec{x},
$$

where the reaction force $\lambda \vec{x}$ is determined from the condition that the trajectory $(\vec{x}(t), \vec{p}(t))$ satisfies the constraints $\phi_{1}=1, \phi_{2}=0$. The system is completely integrable due to the linear first integral $f=\langle\vec{b}, \vec{x} \times \vec{p}+\epsilon \vec{x}\rangle$. 
Generalization to adjoint orbits. The natural generalization of the magnetic spherical pendulum to the orbit $\mathcal{O}(a)$ is the natural mechanical system with the kinetic energy given by the normal metric $d s_{0}^{2}$ and the potential function $V(x)=-\langle b, x\rangle$, i.e., with Hamiltonian

$$
H(x, p)=\frac{1}{2}\langle[x, p],[x, p]\rangle-\langle b, x\rangle,
$$

under the influence of the magnetic force field given by $\epsilon \Omega_{\mathrm{KK}}$, where $\Omega_{\mathrm{KK}}$ is the standard Kirillov-Kostant form. Similar systems on the complex projective spaces are studied in [32].

Proposition 2. The equations of the magnetic pendulum, in redundant variables $(x, p)$, are given by

$$
\begin{aligned}
\dot{x} & =[x,[p, x]], \\
\dot{p} & =[p,[p, x]]+\epsilon[x, p]+b-\operatorname{pr}_{\operatorname{ann}(x)} b,
\end{aligned}
$$

Proof. The equations of the geodesic flow of the normal metric are derived in [3] (see also [9]). The flow is given by the equations:

$$
\begin{aligned}
\dot{x} & =[x,[p, x]], \\
\dot{p} & =[p,[p, x]] .
\end{aligned}
$$

The magnetic and potential forces have no influence to the equation (21), while the second equation takes the form

$$
\dot{p}=[p,[p, x]]+b+\Pi+\Lambda,
$$

where the Lagrange multiplier $\Lambda \in \operatorname{ann}(x)$ is determined from the condition that the trajectory $(x(t), p(t))$ belongs to $T^{*} \mathcal{O}(a)$. On the other side, the magnetic force $\Pi$ is equal to $\epsilon[x, p]$. For example, one can get this relation considering the magnetic geodesic flow of the normal metric (i.e., $V(x) \equiv 0$ ) and the conservation of the shifted momentum map

$$
\begin{aligned}
\dot{\Phi}_{\epsilon}=[x, \dot{p}]+[\dot{x}, p]+\epsilon \dot{x}=[x,[p,[p, x]]+\Pi+\Lambda] \\
+[[x,[p, x]], p]+\epsilon[x,[p, x]]=[x, \Pi+\epsilon[p, x]]=0 .
\end{aligned}
$$

In order to find $\Lambda$, take the (local) base $e_{1}(\tilde{x}), \ldots, e_{r}(\tilde{x})$ of ann $(\tilde{x})\left(\left[e_{i}(\tilde{x}), \tilde{x}\right]=0\right)$, which is orthonormal at $\tilde{x}=x$. Then $\Lambda=\sum_{i=1}^{r} \lambda_{i} e_{i}(x)$. The Lagrange multipliers $\lambda_{i}$ are determined from the conditions

$$
\frac{d}{d t}\left\langle p, e_{i}(x)\right\rangle=\left\langle\dot{p}, e_{i}(x)\right\rangle+\left\langle p, \dot{e}_{i}(x)\right\rangle=0, \quad i=1, \ldots, r
$$


From the identity $\left[e_{i}(\tilde{x}), \tilde{x}\right] \equiv 0$ we have $\left[\dot{e}_{i}(x), x\right]+\left[e_{i}(x), \dot{x}\right]=\left[\dot{e}_{i}(x), x\right]+$ $\left[e_{i}(x),[x,[p, x]]\right]$. On the other side, the Jacobi identity gives $\left[e_{i}(x),[x,[p, x]]\right]=$ $\left[\left[[p, x], e_{i}(x)\right], x\right]$. Therefore

$$
\dot{e}_{i}(x)+\left[[p, x], e_{i}(x)\right] \in \operatorname{ann}(x), \quad i=1, \ldots, r .
$$

Finally, combining (23), (24) and (25), we get $\lambda_{i}=-\left\langle b, e_{i}(x)\right\rangle$, i.e,

$$
\Lambda=-\operatorname{pr}_{\mathrm{ann}(x)} b .
$$

This proves (20).

\section{Integrability of magnetic pendulum}

By the use of the momentum mapping $\Phi_{\epsilon}$, the equations of motion of the magnetic pendulum on the orbit $\mathcal{O}(a)$, can be rewritten in the symmetric form

$$
\begin{aligned}
\dot{x} & =\left[\Phi_{\epsilon}, x\right], \\
\dot{\Phi}_{\epsilon} & =[x, b] .
\end{aligned}
$$

The above system can be naturally understood via another representation of $\left(T^{*} \mathcal{O}(a), \omega+\epsilon \rho^{*} \Omega_{\mathrm{KK}}\right)$, as a coadjoint orbit in the dual space of the semidirect product $\mathfrak{g} \oplus_{\mathrm{ad}} \mathfrak{g}$.

Realization of $T^{*} \mathcal{O}(\boldsymbol{a})$ in $\left(\mathfrak{g} \oplus_{\mathrm{ad}} \mathfrak{g}\right)^{*}$. To prove the complete integrability below, we shall introduce the semidirect product $\mathfrak{g} \oplus_{\text {ad }} \mathfrak{g}$ in a slightly unusual way, by the use of contraction of Lie algebras.

From now on we suppose that $G$ is a compact semisimple Lie group. Then for $\langle\cdot, \cdot\rangle$ we can take the Killing form multiplied by -1 . Let $\mathfrak{g}^{\mathbb{C}}=\mathfrak{g} \otimes \mathbb{C}$. Then $\mathfrak{g}^{\mathbb{C}}$ is a semisimple complex Lie algebra. Denote by $\mathfrak{g}_{0}$ the real semisimple Lie algebra obtained from $\mathfrak{g}^{\mathbb{C}}$ :

$$
\mathfrak{g}_{0}=\mathfrak{g} \oplus i \mathfrak{g}, \quad i^{2}=-1 .
$$

Then $\operatorname{dim} \mathfrak{g}_{0}=\operatorname{dim}_{\mathbb{R}} \mathfrak{g}^{\mathbb{C}}=2 \operatorname{dim}_{\mathbb{C}} \mathfrak{g}^{\mathbb{C}}=2 \operatorname{dim} \mathfrak{g}$ and

$$
\operatorname{rank}_{\mathbb{C}} \mathfrak{g}^{\mathbb{C}}=\operatorname{rank} \mathfrak{g}=r, \quad \operatorname{rank} \mathfrak{g}_{0}=2 \operatorname{rank} \mathfrak{g}=2 r .
$$

Let $p_{1}, \ldots, p_{r}$ be the set of basic homogeneous invariant polynomials on $\mathfrak{g}$ considered as complex invariant polynomials on $\mathfrak{g}^{\mathbb{C}}$. Then their real and imaginary parts form a set of basic polynomial invariants on $\mathfrak{g}_{0}$.

The real algebra $\mathfrak{g}_{0}$ has the symmetric pair decomposition: $\mathfrak{g}_{0}=\mathfrak{g}+i \mathfrak{g}$ :

$$
[\mathfrak{g}, \mathfrak{g}] \subset \mathfrak{g}, \quad[\mathfrak{g}, i \mathfrak{g}] \subset i \mathfrak{g}, \quad[i \mathfrak{g}, i \mathfrak{g}] \subset \mathfrak{g},
$$


and one can consider the contraction of $\mathfrak{g}_{0}$ : the real Lie algebra $\mathfrak{g}_{\theta}$ with the same linear space as $\mathfrak{g}_{0}$ and the Lie bracket defined by

$$
\left[\xi_{1}+i \eta_{1}, \xi_{2}+i \eta_{2}\right]_{\theta}=\left[\xi_{1}, \xi_{2}\right]+i\left[\eta_{1}, \xi_{2}\right]+i\left[\xi_{1}, \eta_{2}\right], \quad \xi_{i}, \eta_{i} \in \mathfrak{g} .
$$

It is clear that $\mathfrak{g}_{\theta}$ is the semidirect product $\mathfrak{g} \oplus_{\text {ad }} i \mathfrak{g}$, where the second term $i \mathfrak{g}$ is considered as a commutative subalgebra.

Now, identify $\mathfrak{g}_{\theta}^{*}$ with $\mathfrak{g}_{\theta}$ and $\mathfrak{g}_{0}^{*}$ with $\mathfrak{g}_{0}$ by means of nondegenerate scalar product

$$
\left(\xi_{1}+i \eta_{1}, \xi_{2}+i \eta_{2}\right)=\left\langle\xi_{1}, \xi_{2}\right\rangle-\left\langle\eta_{1}, \eta_{2}\right\rangle
$$

which is proportional to the Killing form of $\mathfrak{g}_{0}$. Then the differential of a smooth function $f$ on $\mathfrak{g}_{0}\left(\right.$ or $\left.\mathfrak{g}_{\theta}\right)$ is $\left.\nabla f\right|_{\xi+i \eta}=\nabla_{\xi} f-i \nabla_{\eta} f$ and the Lie-Poisson brackets on $\mathfrak{g}_{0}$ and $\mathfrak{g}_{\theta}$ become

$$
\begin{aligned}
\{f, g\}_{\mathfrak{g}_{0}}(\xi+i \eta) & =\left(\xi+i \eta,\left[\nabla_{\xi} f-i \nabla_{\eta} f, \nabla_{\xi} g-i \nabla_{\eta} g\right]\right) \\
& =\left\langle\xi,\left[\nabla_{\xi} f, \nabla_{\xi} g\right]-\left[\nabla_{\eta} f, \nabla_{\eta} g\right]\right\rangle+\left\langle\eta,\left[\nabla_{\xi} f, \nabla_{\eta} g\right]+\left[\nabla_{\eta} f, \nabla_{\xi} g\right]\right\rangle, \\
\{f, g\}_{\mathfrak{g}_{\theta}}(\xi+i \eta) & =\left(\xi+i \eta,\left[\nabla_{\xi} f-i \nabla_{\eta} f, \nabla_{\xi} g-i \nabla_{\eta} g\right]_{\theta}\right) \\
& =\left\langle\xi,\left[\nabla_{\xi} f, \nabla_{\xi} g\right]\right\rangle+\left\langle\eta,\left[\nabla_{\xi} f, \nabla_{\eta} g\right]+\left[\nabla_{\eta} f, \nabla_{\xi} g\right]\right\rangle .
\end{aligned}
$$

Note that a generic symplectic leaf (coadjoint orbit) in $\left(\mathfrak{g}_{\theta},\{\cdot, \cdot\}_{\mathfrak{g}_{\theta}}\right)$ has the same dimension as the orbit in $\mathfrak{g}_{0}$, that is $2 \operatorname{dim} \mathfrak{g}-2 r$ (see [10]).

It is well known that the cotangent bundle to the orbit of the linear representation of a Lie group to the vector space can be seen as a coadjoint orbit in the dual space of the semidirect of the group with the vector space (e.g., see [16]). The similar statement holds for the magnetic cotangent bundles:

Proposition 3. The mapping $\Theta_{\epsilon}: T^{*} \mathcal{O}(a) \rightarrow\left(\mathfrak{g} \oplus_{\mathrm{ad}} i \mathfrak{g}\right)^{*}$ given by

$$
\Theta_{\epsilon}(x, p)=\Phi_{\epsilon}(x, p)+i x
$$

is a symplectomorphism between $T^{*} \mathcal{O}($ a) endowed with the twisted symplectic form $\omega+\epsilon \rho^{*} \Omega_{\mathrm{KK}}$ and the coadjoint orbit of the element $\epsilon a+i$ in $\left(\mathfrak{g} \oplus_{\mathrm{ad}} i \mathfrak{g}\right)^{*}$ endowed with the canonical Kirillov-Kostant symplectic form.

Novikov and Schmeltzer have constucted such mapping for the orbits of coadjoint representation of the three-dimensional Euclidean space motion group which are symplectomorphic to the magnetic cotangent bundles of the sphere (see [26]). This problem is further developed in [19], [13], [30], [2].

Proof. We use the following general statement. Suppose that we have a symplectic manifold $M$ endowed with a transitive Hamiltonian action of a certain Lie group $K$. Consider the corresponding momentum mapping $\Theta: M \rightarrow \mathfrak{k}^{*}$. Then from the standard properties of a momentum mapping it follows that 
1) the image of $\Theta$ is a single coadjoint orbit $O \subset \mathfrak{k}^{*}$,

2) $\Theta: M \rightarrow O$ is a symplectic covering.

In our case we just need to describe the transitive Hamiltonian action of the semidirect product $G \times{ }_{\mathrm{Ad}} i \mathfrak{g}$ on the cotangent bundle $T^{*} \mathcal{O}(a)$. Such an action exists and is very natural. Indeed, let us consider first the standard action of $G$ on the cotangent bundle $T^{*} O(a)$. As we saw above, the corresponding momentum mapping is exactly $\Phi_{\epsilon}$. Now we extend this action by adding the following action of the vector space $\mathfrak{g}$ :

$$
\eta \cdot(x, p)=\left(x, p+\operatorname{pr}_{\mathrm{ann}(x)^{\perp}}(\eta)\right), \quad \eta \in \mathfrak{g},
$$

where $x \in \mathcal{O}, p \in T_{x}^{*} \mathcal{O}(a)=\operatorname{ann}(x)^{\perp}$. Whence the action of the whole semidirect product $G \times \operatorname{Ad} i \mathfrak{g}$ on $T^{*} \mathcal{O}(a)$ is given by:

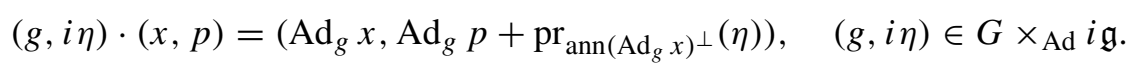

It is easy to verify that this formula defines an action and this action is Hamiltonian. We know this for the first component, and the action of the second one (30) is generated by translations along the Hamiltonian vector fields of the functions

$$
H_{\eta}(x)=-\langle x, \eta\rangle \text {. }
$$

(Notice that this flow is the same for all structures $\omega+\epsilon \rho^{*} \Omega_{\mathrm{KK}}, \epsilon \in \mathbb{R}$ ).

Thus the Hamiltonian with respect to $\omega+\epsilon \rho^{*} \Omega_{\mathrm{KK}}$ corresponding to an element $\xi+i \eta \in \mathfrak{g} \oplus_{\mathrm{ad}} i \mathfrak{g}$ takes the form:

$$
\begin{aligned}
H_{\xi+i \eta}(x, p) & =H_{\xi}(x, p)+H_{\eta}(x, p) \\
& =\left\langle\Phi_{\epsilon}(x, p), \xi\right\rangle-\langle x, \eta\rangle=\left(\Phi_{\epsilon}(x, p)+i x, \xi+i \eta\right)
\end{aligned}
$$

This implies that the formula for the momentum mapping is given by (29), where we use the natural identification of $\left(\mathfrak{g} \oplus_{\mathrm{ad}} i \mathfrak{g}\right)^{*}$ and $\mathfrak{g} \oplus_{\mathrm{ad}} i \mathfrak{g}$ by means of the scalar product (28).

Since the above action is obviously transitive, we conclude that $\Theta_{\epsilon}$ is a symplectic covering over a certain coadjoint orbit. It is not hard to verify that $\Theta_{\epsilon}$ is one-to-one with the image, and therefore is a global symplectomorphism, as required.

Compatible Poisson brackets and integrability. Since the Hamiltonian flow

$$
\dot{f}=\{f, h\}_{\mathfrak{g}_{\theta}}
$$

of $h(\xi+i \eta)=\frac{1}{2}\langle\xi, \xi\rangle-\langle b, \eta\rangle$ is given by

$$
\frac{d}{d t}(\xi+i \eta)=[\eta, b]+i[\xi, \eta],
$$


from Proposition 3 we reobtain the equations (26), (27).

The flow (31) is completely integrable. This is related to the general construction of integrable systems by the use of symmetric pair decompositions of Lie algebras and compatibility of Poisson brackets $\{\cdot, \cdot\}_{\mathfrak{g}_{0}},\{\cdot, \cdot\}_{\mathfrak{g}_{\theta}}$ and $\{\cdot, \cdot\}_{i b}$, where

$$
\begin{aligned}
\{f, g\}_{i b}(\xi+i \eta) & =\left(i b,\left[\nabla_{\xi} f-i \nabla_{\eta} f, \nabla_{\xi} g-i \nabla_{\eta} g\right]\right) \\
& =\left\langle b,\left[\nabla_{\xi} f, \nabla_{\eta} g\right]+\left[\nabla_{\eta} f, \nabla_{\xi} g\right]\right\rangle
\end{aligned}
$$

(Reyman [29], see also [5]).

Let $\mathfrak{A}$ be the algebra of linear functions on $\operatorname{ann}(b)$, lifted to the linear functions on $\mathfrak{g}_{\theta}$ :

$$
\mathfrak{A}=\left\{f_{\mu}(\xi+i \eta)=\langle\mu, \xi\rangle, \mu \in \operatorname{ann}(b)\right\}
$$

Further, let

$$
\mathfrak{B}=\left\{\mathfrak { R e } \left(p_{j}\left(\lambda \xi+i\left(\eta+\lambda^{2} b\right)\right), \mathfrak{I m}\left(p_{j}\left(\lambda \xi+i\left(\eta+\lambda^{2} b\right)\right), \lambda \in \mathbb{R}, j=1, \ldots, r\right\},\right.\right.
$$

where $p_{j}$ are basic invariant polynomials of the Lie algebra $\mathfrak{g}^{\mathbb{C}}$. Then $\mathfrak{B}$ is commutative, and $\mathfrak{A}+\mathfrak{B}$ is a complete (non-commutative) set of integrals of (31) (see [4] or Theorem 1.5 in [5]).

From Proposition 3 we get

Corollary 2. The magnetic pendulum system (19), (20) is completely integrable on a generic orbit $\mathcal{O}(a)$. The complete set of integrals is

$$
\Theta_{\epsilon}^{*}(\mathfrak{A}+\mathfrak{B})=\left\{f\left(\Phi_{\epsilon}(x, p)+i x\right), f \in \mathfrak{A}+\mathfrak{B}\right\} .
$$

Note that we can always construct a complete commutative subalgebra in $\mathfrak{A}$ (more precisely, in the symmetric algebra of $\mathfrak{A}$, i.e. the polynomial algebra generated by linear functions $f_{\mu} \in \mathfrak{A}$ ). Thus we have complete commutative integrability. In particular, if $b$ is a regular element of $\mathfrak{g}$, then $\mathfrak{A}$ is commutative and contained in $\mathfrak{B}$.

The integrability of the system (31) on the whole phase space $\mathfrak{g}_{\theta}$ do not implies directly the integrability on singular orbits. As usual (see, for instance [22], [11], [5]), to prove the completeness of integrals on singular orbits, some additional analysis has to be done.

Let $\mathcal{O}(a)$ be an arbitrary orbit.

Theorem 4. For a generic regular element $b \in \mathfrak{g}$, the magnetic pendulum system on ( $\left.T^{*} \mathcal{O}(a), \omega+\epsilon \rho^{*} \Omega_{\mathrm{KK}}\right)$, described by equations (19), (20), is completely integrable. The complete commutative set of integrals is

$\Theta_{\epsilon}^{*} \mathfrak{B}=\left\{\mathfrak{R e}\left(p_{j}\left(\lambda[x, p]+\epsilon x+i\left(x+\lambda^{2} b\right)\right), \mathfrak{I m}\left(p_{j}\left(\lambda[x, p]+\epsilon x+i\left(x+\lambda^{2} b\right)\right)\right\}\right.\right.$. 
Proof. $\Theta_{\epsilon}^{*} \mathfrak{B}$ is complete on $\left(T^{*} \mathcal{O}(a), \omega+\epsilon \rho^{*} \Omega_{\mathrm{KK}}\right)$ if and only if $\mathfrak{B}$ is complete on the coadjoint orbit $\Theta_{\epsilon}\left(T^{*} \mathcal{O}(a)\right)$.

Consider the pencil of compatible Poisson structures

$$
\Lambda_{\lambda_{1}, \lambda_{2}}=\lambda_{1}\{\cdot, \cdot\}_{\mathfrak{g}_{\theta}}+\lambda_{2}\left(\{\cdot, \cdot\}_{\mathfrak{g}_{0}}+\{\cdot, \cdot\}_{i b}\right), \quad \lambda_{1}, \lambda_{2} \in \mathbb{R}, \lambda_{1}^{2}+\lambda_{2}^{2} \neq 0 .
$$

The functions (32) are Casimir functions for $\Lambda_{\lambda_{1}, \lambda_{2}}$, where $\lambda_{1}+\lambda_{2} \neq 0, \lambda_{2} \neq 0$, $\lambda=\sqrt{\lambda_{2} /\left(\lambda_{2}+\lambda_{1}\right)}$ [29]. Since we deal with analytic functions, we only need to prove the completeness of $\mathscr{B}$ at one point in $\Theta_{\epsilon}\left(T^{*} \mathcal{O}(a)\right)$. Also, the completeness of $\mathscr{B}$ for one element $b$ implies the completeness for a generic $b \in \mathfrak{g}$.

Consider the point $\epsilon a+i a \in \Theta_{\epsilon}\left(T^{*} \mathcal{O}(a)\right)$ and take an arbitrary regular element $b \in \operatorname{ann}(a)$. According Theorem 1.1 [5], $\mathscr{B}$ is complete at $\epsilon a+i a$ with respect to the Poisson bracket $\Lambda_{1,0}=\{\cdot, \cdot\}_{\mathfrak{g}_{\theta}}$ if and only if

(A1) $\quad \operatorname{rank} \Lambda_{\lambda_{1}, \lambda_{2}}=2 \operatorname{dim} \mathfrak{g}-2 r$ for all $\left(\lambda_{1}, \lambda_{2}\right) \neq(1,0)$;

(A2) $\operatorname{dim}\left\{\xi+i \eta \in \operatorname{ker} \Lambda_{1,0} \mid \Lambda_{0,1}\left(\xi+i \eta, \operatorname{ker} \Lambda_{1,0}\right)=0\right\}=2 r$.

Here the Poisson brackets $\Lambda_{\lambda_{1}, \lambda_{2}}$ are taken at the point $\epsilon a+i a$ and they are considered as skew-symmetric bilinear forms on $\mathfrak{g}_{\theta}$. Furthermore, all objects are assumed to be (again) complexified.

Since $b$ is a regular element in $\mathfrak{g}$, it follows that $i b$ is a regular element of the semisimple Lie algebra $\mathfrak{g}_{0}$ :

$$
\operatorname{ann}_{\mathfrak{g} 0}(i b)=\operatorname{ann}_{\mathfrak{g}}(b)+i \operatorname{ann}_{\mathfrak{g}}(b) .
$$

$\left(\operatorname{ann}_{\mathfrak{g}}(b)\right.$ is a maximal commutative subalgebra of $\mathfrak{g}$.)

The condition (A1), for $\lambda_{1}+\lambda_{2} \neq 0$ is equivalent to the regularity of the elements $\lambda \epsilon a+i a+\lambda^{2} i b, \lambda \in \mathbb{C}, \lambda \neq 0$, in the Lie algebra $\mathfrak{g}_{0}$. This follows easily from the regularity of $i b$.

Now, consider the skew-symmetric form $\Lambda_{-1,1}$. We have

$$
\begin{aligned}
\Lambda_{-1,1}\left(\xi_{1}-i \eta_{1}, \xi_{2}-i \eta_{2}\right)=- & \left(\epsilon a+i a,\left[\xi_{1}-i \eta_{1}, \xi_{2}-i \eta_{2}\right]_{\theta}\right) \\
& +\left(\epsilon a+i a,\left[\xi_{1}-i \eta_{1}, \xi_{2}-i \eta_{2}\right]\right) \\
& +\left(i b,\left[\xi_{1}-i \eta_{1}, \xi_{2}-i \eta_{2}\right]\right) \\
=- & \left\langle\epsilon a,\left[\eta_{1}, \eta_{2}\right]\right\rangle+\left\langle b,\left[\xi_{1}, \eta_{2}\right]+\left[\eta_{1}, \xi_{2}\right]\right\rangle
\end{aligned}
$$

Therefore, $\xi-i \eta \in \operatorname{ker} \Lambda_{-1,1}$ if and only if

$$
[b, \eta]=0, \quad[\xi, b]-[\eta, \epsilon a]=0 .
$$

The first equation yields $\eta \in \operatorname{ann}_{\mathfrak{g}}(b)$. On the other hand, since $b \in \operatorname{ann}_{\mathfrak{g}}(a)$, we have $\left[\operatorname{ann}_{\mathfrak{g}}(b), a\right]=0$. Thus, the second equation reduces to $[\xi, b]=0$, i.e., $\xi \in \operatorname{ann}_{\mathfrak{g}}(b)$ and

$$
\operatorname{dim} \operatorname{ker} \Lambda_{-1,1}=2 r \text {. }
$$


It remains to verify (A2). We have

$$
\begin{aligned}
\Lambda_{1,0}\left(\xi_{1}-i \eta_{1}, \xi_{2}-i \eta_{2}\right) & =\left(\epsilon a+i a,\left[\xi_{1}-i \eta_{1}, \xi_{2}-i \eta_{2}\right]_{\theta}\right) \\
& =\left\langle\epsilon a,\left[\xi_{1}, \xi_{2}\right]\right\rangle+\left\langle a,\left[\xi_{1}, \eta_{2}\right]+\left[\eta_{1}, \xi_{2}\right]\right\rangle
\end{aligned}
$$

Similarly as above $\xi-i \eta$ belongs to $\operatorname{ker} \Lambda_{1,0}$ if and only if $[\xi, a]=0,[\eta, a]=0$, i.e.,

$$
\operatorname{ker} \Lambda_{1,0}=\operatorname{ann}_{\mathfrak{g}}(a)+i \operatorname{ann}_{\mathfrak{g}}(a)
$$

We need to find the dimension of the space

$$
\begin{aligned}
K & =\left\{\xi-i \eta \in \operatorname{ker} \Lambda_{1,0} \mid \Lambda_{0,1}\left(\xi-i \eta, \operatorname{ker} \Lambda_{1,0}\right)=0\right\} \\
& =\left\{\xi-i \eta \in \operatorname{ker} \Lambda_{1,0} \mid\left(\epsilon a+i a+i b,\left[\xi-i \eta, \operatorname{ann}_{\mathfrak{g}}(a)+i \operatorname{ann}_{\mathfrak{g}}(a)\right]\right)=0\right\} \\
& =\left\{\xi-i \eta \in \operatorname{ker} \Lambda_{1,0} \mid\left(i b,\left[\xi-i \eta, \operatorname{ann}_{\mathfrak{g}}(a)+i \operatorname{ann}_{\mathfrak{g}}(a)\right]\right)=0\right\}
\end{aligned}
$$

Whence, $K$ consists of those elements in $\operatorname{ann}_{\mathfrak{g}}(a)+i \operatorname{ann}_{\mathfrak{g}}(a)$ which commute with $i b$. But, since $i b$ is a regular element of $\mathfrak{g}_{0}$, we find $\operatorname{dim} K=2 r$. The theorem is proved.

Remark 4. From Corollary 2, by taking $b=0$, we get complete integrability of the magnetic geodesic flows of normal metrics on regular orbits $\mathcal{O}(a)$. It is interesting that in this case we have

$$
\mathcal{F}_{1}^{\epsilon}=\Theta_{\epsilon}^{*} \mathfrak{A},
$$

and $\Theta_{\epsilon}^{*} \mathfrak{B}$ coincides with the set of commuting $G$-invariant functions on $T^{*} \mathcal{O}(a)$ obtained by shifting of argument (16):

$$
\mathscr{B}_{a}=\Theta_{\epsilon}^{*} \mathfrak{B}
$$

In this sense, remarkably, the shifting of argument method [24] can be seen as a particular case of the method of contraction of Lie algebras [4], [10]. By modifying the proof of Theorem 4, it would be possible to give a proof of the completeness of $\mathcal{B}_{a}+\mathcal{F}_{1}^{0}$ on singular orbits $\mathcal{O}(a)$, different from those given in [8], [23].

Remark 5. As it follows from Reyman and Semenov-Tian-Shanski [30] (or by straightforward computation) the equations (26), (27) are equivalent to the L-A pair

$$
\dot{L}(\lambda)=[L(\lambda), A(\lambda)]
$$

with a spectral parameter $\lambda$, where $L(\lambda)=\lambda \Phi_{\epsilon}+i\left(x+\lambda^{2} b\right), A(\lambda)=\Phi_{\epsilon}+i \lambda b$. The integrals $\Theta_{\epsilon}^{*} \mathfrak{B}$ are exactly the integrals arising from the L-A representation of the system. 


\section{References}

[1] R. Abraham and J. E. Marsden, Foundations of mechanics. Second edition, Benjamin/Cummings Publishing Co., Inc., Advanced Book Program, Reading, Mass., 1978. Zbl 0393.70001 MR 0515141

[2] P. Baguis, Semidirect products and the Pukanszky condition. J. Geom. Phys. 25 (3-4) (1998), 245-270. Zbl 0932.22011 MR 1619845

[3] A. M. Bloch, R. W. Brockett, and P. E. Crouch, Double Bracket Equations and Geodesic Flows on Symmetric Spaces. Comm. Math. Phys. 187 (1997), 357-373. Zbl 0884.58079 MR 1463833

[4] A. V. Bolsinov, Completely integrable systems on contractions of Lie algebras. Trudy Sem. Vektor. Tenzor. Anal. 22 (1985), 8-16 (in Russian). Zbl 0599.17012 MR 1041777

[5] A. V. Bolsinov, Compatible Poisson brackets on Lie algebras and the completeness of families of functions in involution. Izv. Akad. Nauk SSSR Ser. Mat. 55 (1) (1991), 68-92; English transl. Math. USSR-Izv. 38 (1) (1992), 69-90. Zbl 0744.58030 MR 1130028

[6] A. V. Bolsinov and B. Jovanović, Integrable geodesic flows on homogeneous spaces. Mat. Sb. 192 (7) (2001), 21-40; English transl. Sb. Math. 192 (7-8) (2001), 951-969. Zbl 1035.53115 MR 1861371

[7] A. V. Bolsinov and B. Jovanović, Non-commutative integrability, moment map and geodesic flows. Ann. Global Anal. Geom. 23 (4) (2003), 305-322. Zbl 1022.37038 MR 1972543

[8] A. V. Bolsinov and B. Jovanović, Complete involutive algebras of functions on cotangent bundles of homogeneous spaces. Math. Z 246 (1-2) (2004), 213-236. Zbl 1066.70012 MR 2031454

[9] A. V. Bolsinov and B. Jovanović, Magnetic Geodesic Flows on Coadjoint Orbits. J. Phys. A 39, L247-L252(2006). Zbl 1089.70010 MR 2220360

[10] A. V. Brailov, Some constructions of the complete commutative sets of functions. Trudy Sem. Vektor. Tenzor. Anal. 22 (1985), 17-24 (in Russian). Zbl 0592.58019 MR 1041778

[11] A. V. Brailov, Construction of complete integrable geodesic flows on compact symmetric spaces. Izv. Acad. Nauk SSSR Ser. Mat. 50 (2) (1986), 661-674; English transl. Math. USSR-Izv. 50 (4) (1986), 19-31. Zbl 0638.58011 MR 0864170

[12] E. A. Buldaeva, On integrable geodesic flows on the adjoint orbits of orthogonal groups. Diploma thesis, Dept. of Mathematics and Mechanics, Moscow State University, May 2002 (in Russian).

[13] C. Duval, J. Elhadad, and G. M. Tuynman, Pukanszky's condition and symplectic induction. J. Differential Geometry 36 (1992), 331-348. Zbl 0738.22006 MR 1180386

[14] D. I. Efimov, The magnetic geodesic flows in a homogeneous field on the complex projective space. Siberian Math. J. 45 (3) (2004), 465-474. Zbl 1097.37046 MR 2078716

[15] D. I. Efimov, The magnetic geodesic flows on a homogeneous symplectic manifold. Siberian Math. J. 46 (1) (2005), 83-93. Zbl 1079.37055 MR 2141306

[16] V. Guillemin and S. Sternberg, Symplectic techniques in physics. Cambridge University Press, Cambridge 1984. Zbl 0576.58012 MR 0770935 
[17] G. A. Hedlund, Fuchsian groups and transitive horocycles. Duke Math. J. 2 (1936), 530-542. Zbl 0015.10201 MR 1545946

[18] B. Jovanović, On the Integrability of Geodesic Flows of Submersion Metrics. Lett. Math. Phys. 61 (2002), 29-39. Zbl 1030.53085 MR 1930080

[19] S. A. Kamalin and A. M. Perelomov, Construction of Canonical Coordinates on Polarized Coadjoint Orbits of Lie Grops. Comm. Math. Phys. 97 (1985), 553-568. Zbl 0578.58016 MR 0787118

[20] M. Kummer, On the construction of the reduced phase space of a Hamiltonian system with symmetry. Indiana Univ. Math. J. 30 (1981), 281-291. Zbl 0425.70019 MR 0604285

[21] S. V. Manakov, A remark on the integration of the Eulerian equations of the dynamics of an $n$-dimensional rigid body. Funkcional. Anal. i Priložen. 10 (4) (1976), 93-94; English transl. Functional Anal. Appl. 10 (4) (1976), 328-329. Zbl 0358.70004 MR 0455031

[22] I. V. Mikityuk, Homogeneous spaces with integrable $G$-invariant Hamiltonian flows. Izv. Acad. Nauk SSSR Ser. Mat. 47 (6) (1983), 1248-1262 (in Russian). Zbl 0539.58016 MR 0727754

[23] I. V. Mikityuk and A. Panasyuk, Bi-Poisson structures and integrability of geodesic flows on homogeneous spaces. Transform. Groups 9 (3) (2004), 289-308. Zbl 1050.22027 MR 2079137

[24] A. S. Mishchenko and A. T. Fomenko, Euler equations on finite-dimensional Lie groups. Izv. Acad. Nauk SSSR Ser. Mat. 42 (2) (1978), 396-415; English transl. Math. USSR-Izv. 42 (2) (1978), 371-389. Zbl 0405.58028 MR 0482832

[25] A. S. Mishchenko and A. T. Fomenko, A generalized Liouville method for the integration of Hamiltonian systems. Funkts. Anal. Prilozh. 12 (2) (1978), 46-56; English trans. Funct. Anal. Appl. 12 (1978), 113-121. Zbl 0405.58028 MR 0516342

[26] S. P. Novikov and I. Shmeltzer, Periodic solutions to Kirchoff equations for a free motion of a rigid body in fluid and Lusternik-Shnirelman-Morse extended theory. Funct. Anal. Appl. 15 (1981), 54-66. Zbl 0571.58009 MR 0630339

[27] N. N. Nekhoroshev, Action-angle variables and their generalization. Trudy Moskov. Mat. Obšč. 26 (1972), 181-198; English transl. Trans. Moscow Math. Soc. 26 (1972), 180-198. Zbl 0284.58009 MR 0365629

[28] J.-P. Ortega and T. S. Ratiu, Momentum maps and Hamiltonian reduction. Progr. Math. 222, Birkhäuser Boston, Boston, MA, 2004. Zbl 02061778 MR 2021152

[29] A. G. Reyman, Integrable Hamiltonian systems connected with graded Lie algebras. Zap. Nauchn. Sem. Leningrad. Otdel. Mat. Inst. Steklov. 95 (1980), 3-54; English transl. J. Soviet Math. 19 (1982), 1507-1545. Zbl 0554.70010

[30] A. G. Reyman and M. A. Semenov-Tian-Shanski, Group theoretical methods in the theory of finite dimensional integrable systems. In Dynamical systems VII (V. I.Arnold, S. P. Novikov, eds.), Springer-Verlag, Berlin, Heidelberg 1994, 116-225. Zbl 0796.58017 MR 0922071

[31] S. T. Sadetov, A proof of the Mishchenko-Fomenko conjecture (1981). Dokl. Akad. Nauk 397 (6) (2004), 751-754; English transl. Dokl. Math. 70 (1) (2004), 635-638. MR 2120177

[32] P. Saksida, Integrable anharmonic oscilators on spheres and hyperbolic spaces. Nonlinearity 14 (2001), 977-994. Zbl 1067.37075 MR 1862807 
[33] I. A. Taimanov, An example of the transition from chaos to integrability in magnetic geodesic flows. Mat. Zametki 76 (4) (2004), 632-634; English transl. Math. Notes 76 (2004), 587-589. Zbl 1068.37019 MR 2113040

[34] A. Thimm, Integrable geodesic flows on homogeneous spaces. Ergodic Theory Dynamical Systems 1 (1981), 495-517. Zbl 0491.58014 MR 0662740

[35] N. T. Zung, Torus actions and integrable systems. In Topological methods in the theory of integrable systems, A. V. Bolsinov, A. T. Fomenko, A. A. Oshemkov, eds., Cambridge Scientific Publ., 2006.

Received October 3, 2006

Alexey V. Bolsinov, Department of Mathematical Sciences, Loughborough University, Loughborough, Leicestershire LE11 3TU UK, and

Department of Mechanics and Mathematics, Moscow State University, 119992, Moscow, Russia

E-mail: A.Bolsinov@lboro.ac.uk

Božidar Jovanović, Mathematical Institute SANU, Kneza Mihaila 35, 11000 Belgrade,

Serbia

E-mail: bozaj@mi.sanu.ac.yu 